\title{
Ca Cobaltites as Potential Cathode Materials for Rechargeable Ca Ion Batteries: Theory and Experiment
}

Haesun Park ${ }^{+, \neq}$, Yanjie Cuit, ${ }^{\dagger}$, Sanghyeon Kim ${ }^{+, \bullet}, J$. T. Vaughey ${ }^{t, \bullet}$, and Peter Zapol ${ }^{*},+, \neq$

$\dagger$ †oint Center for Energy Storage Research (JCESR), ¥Materials Science Division, QChemical Sciences and Engineering Division, Argonne National Laboratory, $9700 \mathrm{~S}$ Cass Ave, Lemont, IL 60439

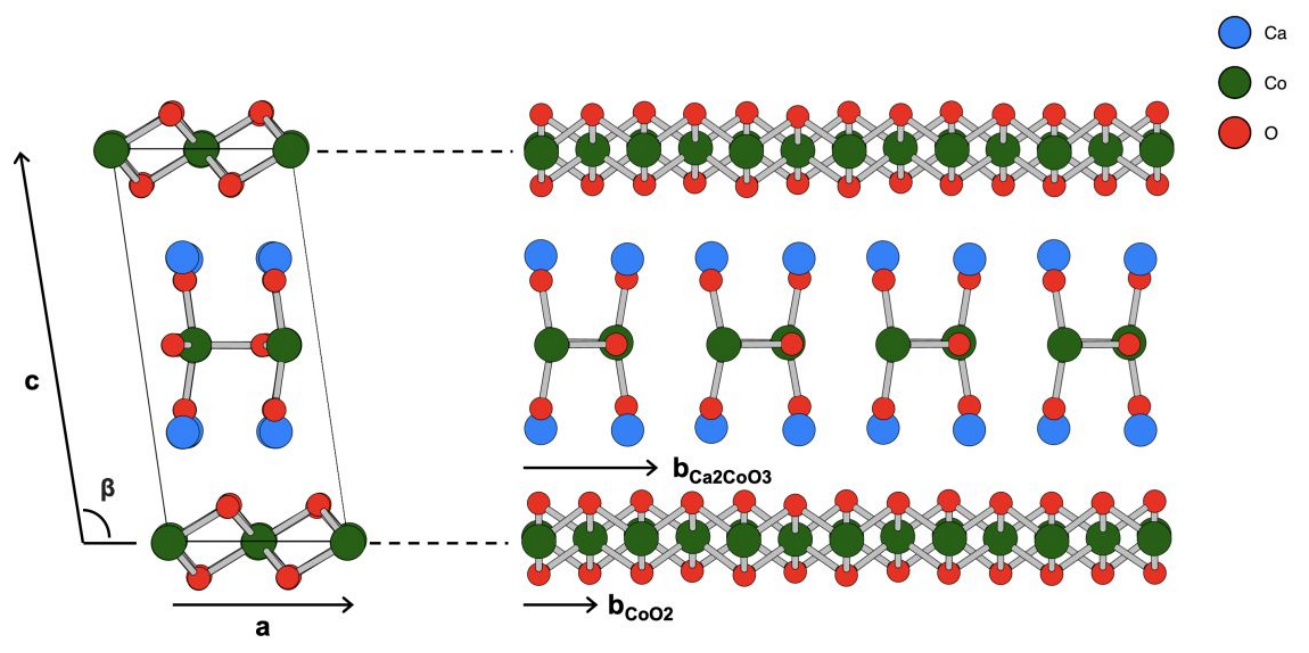

Figure $\mathrm{S} 1$ The snapshot of $\left[\mathrm{Ca}_{2} \mathrm{CoO}_{3}\right]\left[\mathrm{CoO}_{2}\right]_{x}$ crystal structure and the lattice parameters feature the structure.

Table S1. Lattice parameters of $\left[\mathrm{Ca}_{2} \mathrm{CoO}_{3}\right]\left[\mathrm{CoO}_{2}\right]_{x}(x=3 / 2,5 / 3$, and 8/5) calculated by DFT and their deviation from the experimental value.

\begin{tabular}{|c|c|c|c|c|c|c|c|c|c|c|}
\hline & $a(\AA)$ & Dev.(\%) & $b_{\mathrm{Ca}_{2} \mathrm{CoO}_{3}}(\AA)$ & Dev.(\%) & $b_{\mathrm{CoO}_{2}}(\AA)$ & Dev.(\%) & $c(\AA ̊)$ & Dev.(\%) & $\beta\left(^{\circ}\right)$ & Dev.(\%) \\
\hline Exp. & 4.83 & & 4.56 & & 2.82 & & 10.84 & & 98.13 & \\
\hline
\end{tabular}




\begin{tabular}{lcccccccccc}
\hline $3 / 2$ & 4.94 & $2.2 \%$ & 4.84 & $6.1 \%$ & 2.93 & $3.9 \%$ & 10.88 & $0.3 \%$ & 98.21 & $0.1 \%$ \\
$(\mathrm{GGA}+\mathrm{U})$ & & & & & & & & & & \\
$5 / 3$ & 4.90 & $1.4 \%$ & 4.72 & $3.5 \%$ & 2.83 & $0.4 \%$ & 10.82 & $-0.2 \%$ & 98.33 & $0.2 \%$ \\
$(\mathrm{GGA}+\mathrm{U})$ & & & & & & & & & & \\
$8 / 5$ & 4.90 & $1.5 \%$ & 4.58 & $0.5 \%$ & 2.86 & $1.4 \%$ & 10.88 & $0.4 \%$ & 98.27 & $0.1 \%$ \\
\hline
\end{tabular}

(a) $\mathrm{CaCO}_{2} \mathrm{O}_{4}$

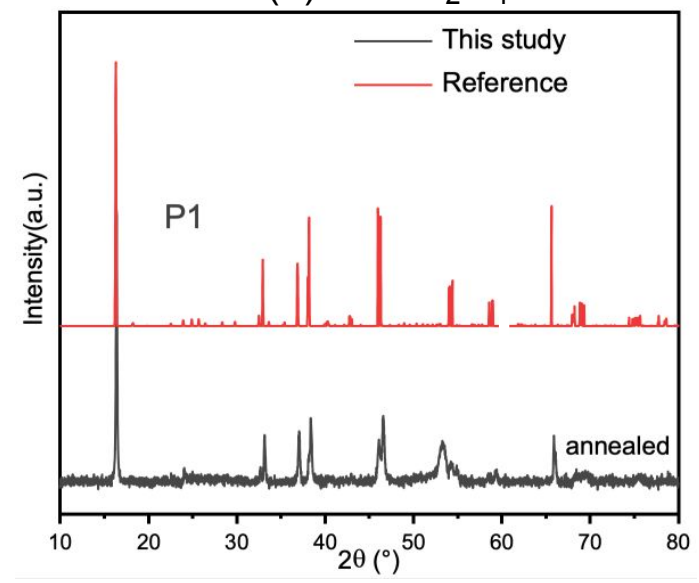

(c) $\left[\mathrm{Ca}_{2} \mathrm{CoO}_{3}\right]\left[\mathrm{CoO}_{2}\right]_{1.62}$

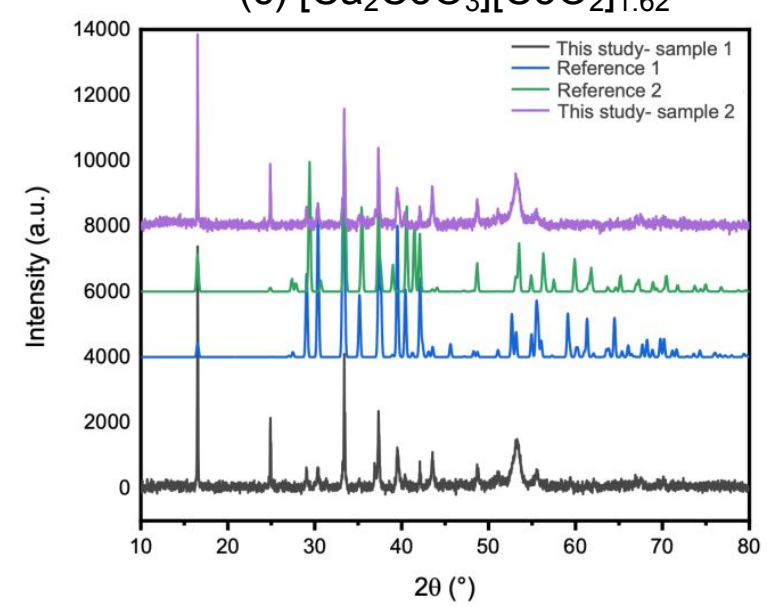

(b) $\mathrm{Ca}_{3} \mathrm{Co}_{2} \mathrm{O}_{6}$

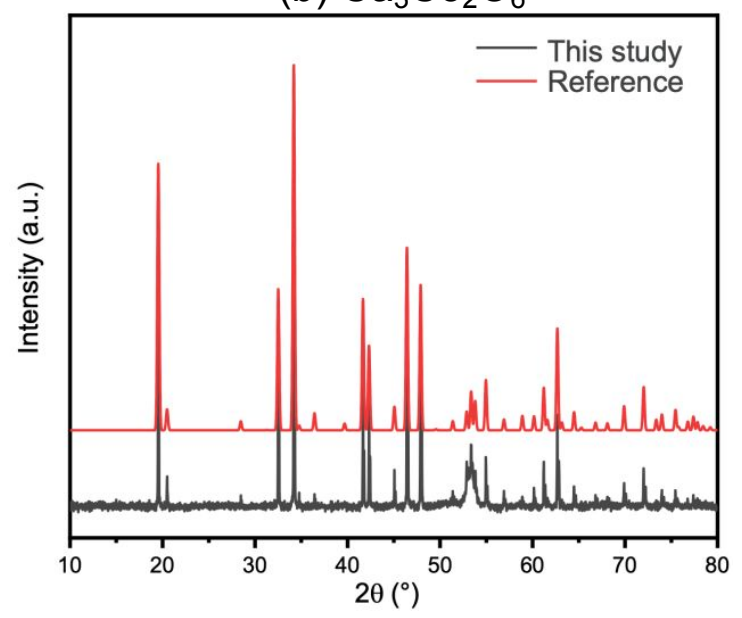

(d) $\mathrm{Ca}_{2} \mathrm{Co}_{2} \mathrm{O}_{5}$

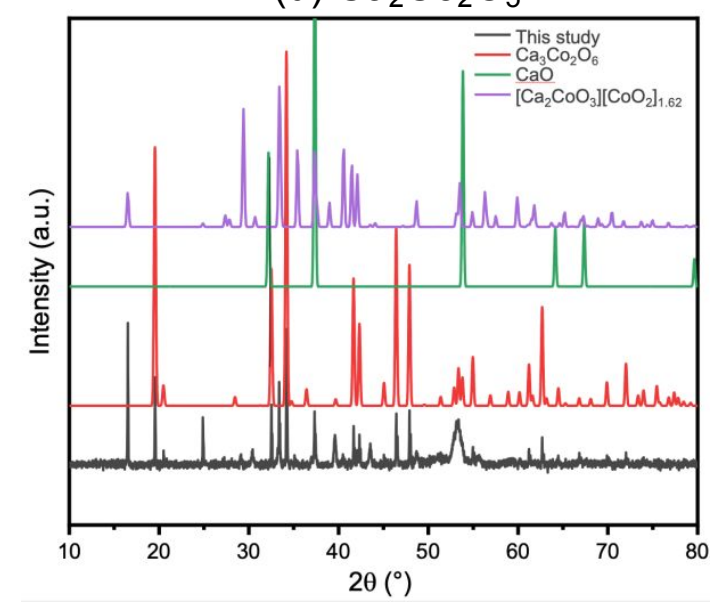

Figure S2. The X-ray diffraction patterns of synthesized samples of (a) $\mathrm{CaCo}_{2} \mathrm{O}_{4}$, (b)

$\mathrm{Ca}_{3} \mathrm{Co}_{2} \mathrm{O}_{6}$, (c) $\left[\mathrm{Ca}_{2} \mathrm{CoO}_{3}\right]\left[\mathrm{CoO}_{2}\right]_{1.62}$, and (d) $\mathrm{Ca}_{2} \mathrm{Co}_{2} \mathrm{O}_{5}$. 
(a) $\mathrm{CaCo}_{2} \mathrm{O}_{4}$

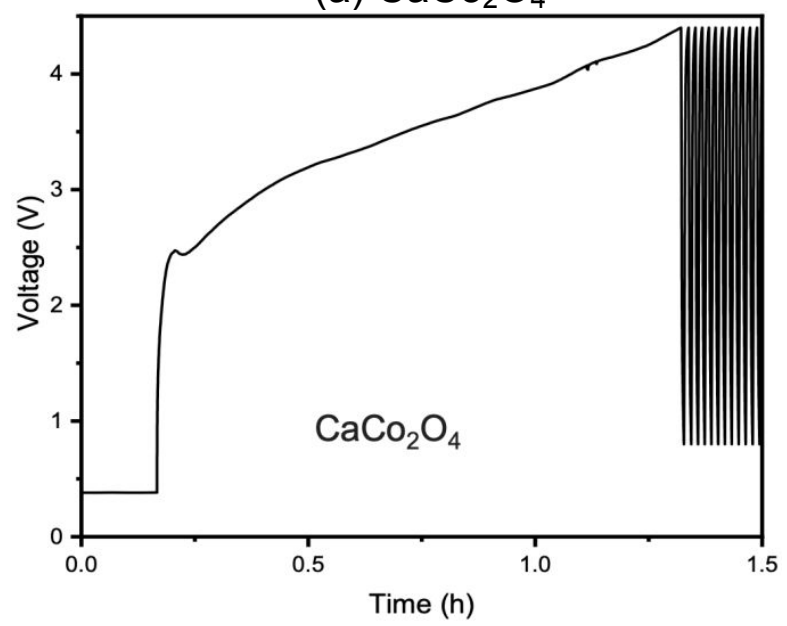

(c) $\left[\mathrm{Ca}_{2} \mathrm{CoO}_{3}\right]\left[\mathrm{CoO}_{2}\right]_{1.62}$

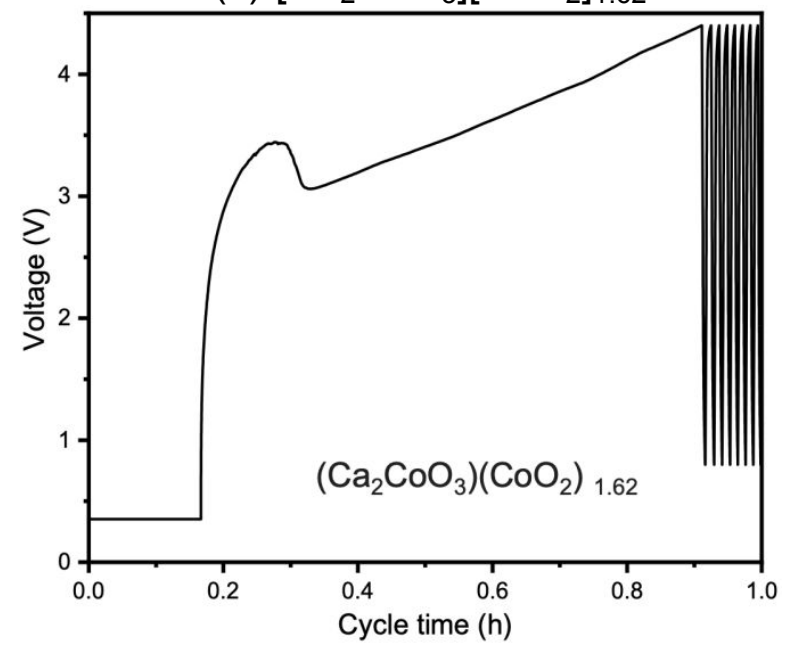

(b) $\mathrm{Ca}_{3} \mathrm{Co}_{2} \mathrm{O}_{6}$

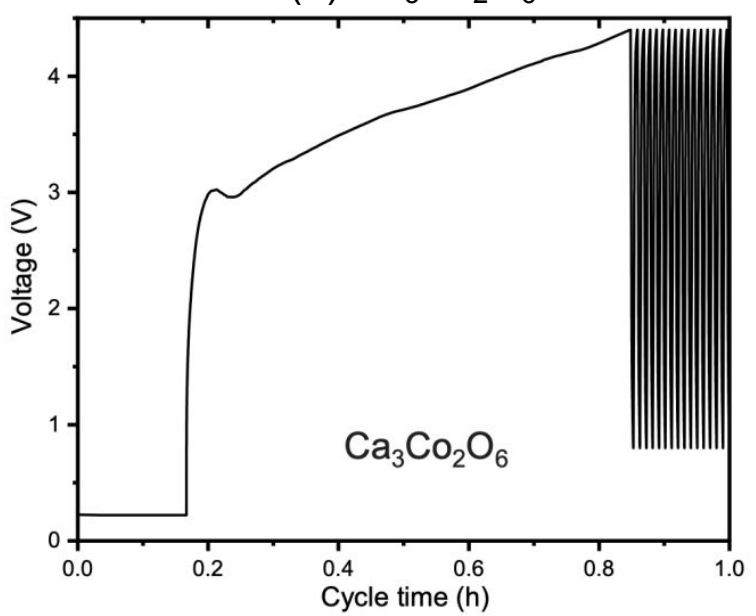


Figure S3. The time vs. voltage curves of (a) $\mathrm{CaCo}_{2} \mathrm{O}_{4}$, (b) $\mathrm{Ca}_{3} \mathrm{Co} 2 \mathrm{O}$, and (c) $\left[\mathrm{Ca}_{2} \mathrm{CoO}_{3}\right]\left[\mathrm{CoO}_{2}\right]_{1.62}$. The small plateau observed at the initial $\mathrm{Ca}$ extraction process represents the extraction of $\mathrm{Ca}$ from the surface of materials.

Table S2. The open circuit voltage (OCV), capacity, and the amount of extracted Ca.

\begin{tabular}{l|ccc}
\hline Compound & OCV (V) & Capacity mAh/g (to 4.2V) & $\begin{array}{c}\text { Extracted Ca } \\
(\%)\end{array}$ \\
\hline $\mathrm{CaCo}_{2} \mathrm{O}_{4}$ & 2.5 & 11 & $\sim 3$ \\
$\mathrm{Ca}_{3} \mathrm{Co}_{2} \mathrm{O}_{6}$ & 3.0 & 7 & $\sim 1.5$ \\
{$\left[\mathrm{Ca}_{2} \mathrm{CoO}_{3}\right]\left[\mathrm{CoO}_{2}\right]_{1.62}$} & 3.5 & 8 & $\sim 2$ \\
\hline
\end{tabular}

Table S3 Synthesis parameters for Ca Cobaltites including precursors and calcination temperatures.

\begin{tabular}{lllll}
\hline Reactants & $\mathrm{CaCO}_{2} \mathrm{O}_{4}$ & $\mathrm{Ca}_{3} \mathrm{Co}_{4} \mathrm{O}_{9}$ & $\mathrm{Ca}_{2} \mathrm{Co}_{2} \mathrm{O}_{5}$ & $\mathrm{Ca}_{3} \mathrm{Co}_{2} \mathrm{O}_{6}$ \\
\hline Mole ratio Ca:Co: $\mathrm{C}_{6} \mathrm{H}_{8} \mathrm{O}_{7} \mathrm{C}_{2} \mathrm{H}_{6} \mathrm{O}_{2}$ & $1: 2: 3: 6$ & $1.5: 2: 3.5:$ & $1: 1: 2: 4$ & $3: 2: 5: 10$ \\
$\mathrm{Ca}\left(\mathrm{NO}_{3}\right)_{2}(1.00 \mathrm{M}) / \mathrm{mL}$ & 10.0 & 15.0 & 15.0 & 30.0 \\
$\mathrm{CO}\left(\mathrm{NO}_{3}\right)_{2}(1.00 \mathrm{M}) / \mathrm{mL}$ & 20.0 & 20.0 & 15.0 & 20.0 \\
$\begin{array}{l}\text { Citric acid }\left(\mathrm{C}_{6} \mathrm{H}_{8} \mathrm{O}_{7}\right) \mathrm{g} \\
\text { Ethylene glycol }\left(\mathrm{C}_{2} \mathrm{H}_{6} \mathrm{O}_{2}\right) \mathrm{g}\end{array}$ & 6.30 & 7.36 & 6.30 & 10.50 \\
$\begin{array}{l}\text { Calcination temperature } \\
\mathrm{h}\end{array}$ & 3.72 & 4.34 & 3.72 & 6.25 \\
\hline
\end{tabular}

